\title{
Sexual Harassment at University of California Libraries: Understanding the Experiences of Library Staff Members
}

\section{Jill Barr-Walker, Courtney Hoffner, Elizabeth McMunn-Tetangco, and Nisha Mody}

In the first study measuring sexual harassment experiences of academic library employees at a single institution, we conducted a census of 1,610 nonstudent employees at the 10-campus University of California Libraries system. This anonymous online survey measured how sexual harassment was experienced and observed in terms of behaviors, exhibitors, reporting and disclosure, institutional support and betrayal, and recommendations for future actions. Out of 579 respondents, 54\% experienced and/or observed sexual harassment at work. Respondents recommended training, workplace culture change, support from leadership, and clear reporting processes in order to address sexual harassment at University of California Libraries.

\section{Introduction}

"Men on my staff are allowed to express strong opinions, even if they are counter to the opinions of our administration. When women have done this, they are shut down immediately and written off. People perpetuate rumors that women are difficult to work with while men are just being who they are."

(Note: The quotes at the beginning of each section are a selection of survey responses from our study.)

In a 2018 pilot study that surveyed library employees at the University of California, San Francisco (UCSF) campus about sexual harassment experiences, 48 percent of respondents indicated that they had experienced sexual harassment at work. ${ }^{1}$ These findings provided the impetus for the current study and correspond to results of a 2018 report by the National Academies of

Jill Barr-Walker is Clinical Librarian at San Francisco General Hospital at the University of California, San Francisco, email: jill.barr-walker@ucsf.edu; Courtney Hoffner is Librarian at the University of California Los Angeles, email: choffner@library.ucla.edu; Elizabeth McMunn-Tetangco is Instruction E Research Librarian at the University of California Merced Library, email: emcmunn@ucmerced.edu; and Nisha Mody is Associate Director at the Network of the National Library of Medicine, Pacific Southwest Region, based at UCLA, email: nmody@library. ucla.edu. (2021 Jill Barr-Walker, Courtney Hoffner, Elizabeth McMunn-Tetangco, and Nisha Mody, AttributionNonCommercial (https://creativecommons.org/licenses/by-nc/4.0/) CC BY-NC. 
Sciences, Engineering, and Medicine (NASEM), which noted that, within academia, more than 50 percent of women faculty and staff and 20 to 50 percent of women students encounter or experience sexual harassment. ${ }^{2}$ Despite NASEM's findings about the prevalence of this issue in academia, sexual harassment of academic library employees has not been comprehensively studied.

The University of California (UC) system comprises 10 campuses within the state of California. Campuses are located in Berkeley, Davis, Irvine, Los Angeles, Merced, Riverside, Santa Barbara, Santa Cruz, San Diego, and San Francisco, locations that are physically and culturally removed from each other. In addition to disparate locations, campuses are different sizes and face different challenges; for example, Berkeley, Los Angeles, and San Diego have large enrollments between 35,000 and 44,000 students, whereas Merced and San Francisco are smaller (between 3,100 and 8,000 students) and are located in rural and urban settings, respectively. Though each UC campus library is a part of the larger UC Libraries system, which has a long tradition of collaboration and sharing, they also operate autonomously in many ways. We wondered if the results of the UCSF pilot study would be similar if extrapolated to the other campuses.

Building on the results of the UCSF pilot, we conducted a research study to understand the scope of this issue across the UC Libraries system. The aim of our study was to explore sexual harassment experiences of nonstudent employees at University of California Libraries. Our research questions included:

- What types of sexual harassment behaviors have UC Libraries employees experienced?

- Who exhibits sexual harassment behaviors to UC Libraries employees?

- What associations exist between sexual harassment experiences and intersectional identities such as race or gender?

- Are there differences in sexual harassment experiences across UC campuses?

- How do UC Libraries employees view their institutions' actions around sexual harassment?

As we explored these questions, our objectives were to:

- Measure sexual harassment experiences qualitatively and quantitatively, including types of behaviors experienced and user categories of those who exhibit behaviors

- Measure opinions of library staff regarding institutional support around sexual harassment

- Produce policy and training recommendations for UC Libraries based on our findings We hoped that by sharing the results of our study, we could begin systemwide conversations about how to address sexual harassment of UC Libraries staff members. While our study design and resulting recommendations were specific to UC Libraries, it is our hope that sharing these results widely will inspire others to begin similar conversations on their campuses.

\section{Literature Review}

"I have felt coerced into giving hugs initiated by a superior."

Despite increased attention worldwide in workplaces, the media, and everyday life, sexual harassment remains a major problem in academia. Headlines, op-ed pieces, social media, and anecdotal conversations underscore both the impact and prevalence of unwanted harassment in multiple spaces, including the workplace. The 2018 NASEM report lists lowered job satis- 
faction and mental and physical withdrawal from work responsibilities as possible outcomes for employees. ${ }^{3}$ People who experience sexual harassment can lose trust in their institutions, especially if they do not perceive that these institutions have done enough to prevent it. In a study using the Institutional Betrayal and Support Questionnaire, graduate students who experienced sexual harassment from faculty or staff members were more likely to report "institutional betrayal" or a lack of support from their institution following their experiences. ${ }^{4}$ Within the library setting, there is some evidence that a certain amount of sexual harassment is normalized. In a 2018 study of academic librarians, 77.4 percent of respondents indicated that they had experienced at least one of the sexual harassment behaviors listed in the survey. ${ }^{5}$ Two anecdotal surveys of librarians showed similar results. In the first survey, 63 percent of public library respondents encountered sexual harassment at work, and the second survey received more than 250 responses to a call asking for librarians to submit stories about sexual harassment experiences. ${ }^{6}$ In the latter survey, story after story of sexual harassment in libraries were provided, which paints a disturbing picture of the types of harassment that library workers experience day in and day out. ${ }^{7}$ Library workers who have made efforts to address sexual harassment of employees at their organizations have suggested the importance of culture change, including actions like developing clear policies and procedures around this issue, organizing bystander training and role-playing exercises for staff to help them feel calm and in control during an experience of harassment, and opening up dialogue between administrators and employees to encourage library workers to speak about this issue. ${ }^{8}$

\section{Methods}

"It hasn't happened in a while but I can say that it has happened a handful of times - always uncalled for and always from people I thought were being friendly at first."

We developed a research protocol that included information about our study design, data analysis techniques, and project plan (see appendix A). To raise awareness of our study and solicit feedback, we shared our protocol with several administrative groups, including: 1) Council of University Librarians (includes the university librarian from each UC campus and the executive director of the California Digital Library); 2) Administrative Services Advisory Group (reports to the Council of University Librarians and advises on matters related to human resources); 3) Librarians Association of the University of California (a statewide organization of all UC librarians); and 4) the CARE Directors Group (sexual violence prevention advocates located on each UC campus). To ensure that we considered the unique needs of each UC campus, we created an Advisory Team, made up of employee representatives from each campus library, to advise on study aims, survey content, dissemination plans, and future actions. Our study was approved by the University of California, San Francisco Institutional Review Board in accordance with the UC Multi-Campus Human Subject Research MOU as exempt research for multi-campus distribution (\#18-25552). ${ }^{9}$

\section{Survey Creation and Distribution}

"I had a supervisor who would direct suggestive comments and jokes toward me, and make comments of a sexual nature concerning my marriage." 
We used REDCap, a secure, web-based survey application, to create and distribute an anonymous online survey to all eligible employees who worked in University of California Libraries $(n=1,610)$ during 6 weeks in November and December 2018. Eligible participants were either 1) a nonstudent employee working in a UC Library that reported to the University Librarian or 2) a member of the Librarians Association of the University of California; other affiliate library employees were not eligible. Because of UC guidelines around mandated reporting, which state that all employees with supervisory responsibilities must report incidents of sexual harassment among UC employees, it was necessary to ensure anonymity of the survey. ${ }^{10}$ We shared the survey via an anonymous link and instructed participants not to share any potentially identifying information, such as names or departments, in write-in questions. To provide further support, we shared contact information for the sexual violence prevention offices (CARE) on each campus within the informed consent form and throughout the survey. All survey questions were optional, and participants were invited to skip questions or stop the survey at any time if they did not feel comfortable.

The survey included six parts: 1) which sexual harassment behaviors participants experienced at UC Libraries; 2) from whom participants experienced sexual harassment behaviors (measured in user categories, such as UC staff, library staff, members of the public); 3) how participants felt their UC campus library and the University of California has supported them with regard to their experience of sexual harassment, using the Institutional Betrayal and Support Questionnaire ${ }^{11}$ and questions about the importance of addressing sexual harassment; 4) how UC Libraries can address sexual harassment in the future; 5) demographic information, including gender and campus; and 6) how participants felt their intersectional identities affect their experiences of sexual harassment (see appendix B).

Sexual harassment behaviors listed in the survey were developed through an iterative process involving feedback from our Advisory team, CARE advocates, and use of an existing scale. ${ }^{12}$ We also included the Institutional Betrayal and Support Questionnaire version 1 (IBSQ.1), which measures feelings around "wrongdoings perpetrated by an institution upon individuals dependent on that institution, including failure to prevent or respond supportively to wrongdoings by individuals (e.g. sexual harassment) committed within the context of the institution." 13

Because of the lack of diversity within libraries, ${ }^{14}$ which is reflected in the population of UC Libraries employees, and the need for maintaining anonymity of participants, we were unable to collect specific demographic information including racial identity and sexual orientation. We believe this information is important to understand the intersectional experiences of library staff members, especially those in underrepresented groups; therefore, we included a question about how participants felt their intersectional identities affect experiences of sexual harassment.

\section{Data Analysis}

"Comments on my appearance have happened from colleagues and from users. It always makes me uncomfortable."

Data cleaning, codebook creation, and quantitative analysis were completed by one author in Microsoft Excel, Google Docs, and Stata (JBW). We assigned each of the four questions 
with write-in responses to a team of two authors to conduct thematic analysis, with each team responsible for two questions. Participant responses were reviewed independently by authors in each team who then shared their ideas about emerging themes, came to consensus on thematic codes, and independently assigned codes to each response. Interrater reliability was conducted, with both authors checking each response and resolving any discrepancies between code assignments via deliberation until resolution. To protect the anonymity of participants, no data beyond aggregate results will be shared.

\section{Results}

We created visual representations of our study results on a website to share with our colleagues, which can be found at https://uclibraries.wixsite.com/shstudy. ${ }^{15}$

\section{Participant Demographics}

"I would share, but I worry that my colleague might see my response in the survey results."

Out of a possible 1,610 respondents, 579 individuals completed the survey (36\% response rate), including 349 women (60\%), 142 men (25\%), 4 of another gender identity (1\%), and 84 people $(14 \%)$ who preferred not to state their gender or left the question blank. Participants represented all UC campuses, with the highest number of respondents from the two largest campuses, UCLA and UC Berkeley (see figure 1). Response rates were highest at campuses with smaller populations, with UC Merced (68\%), UC Santa Cruz (45\%), and UC San Francisco $(45 \%)$ representing the top three. Seventy-five participants (13\%) preferred not to list their campus.

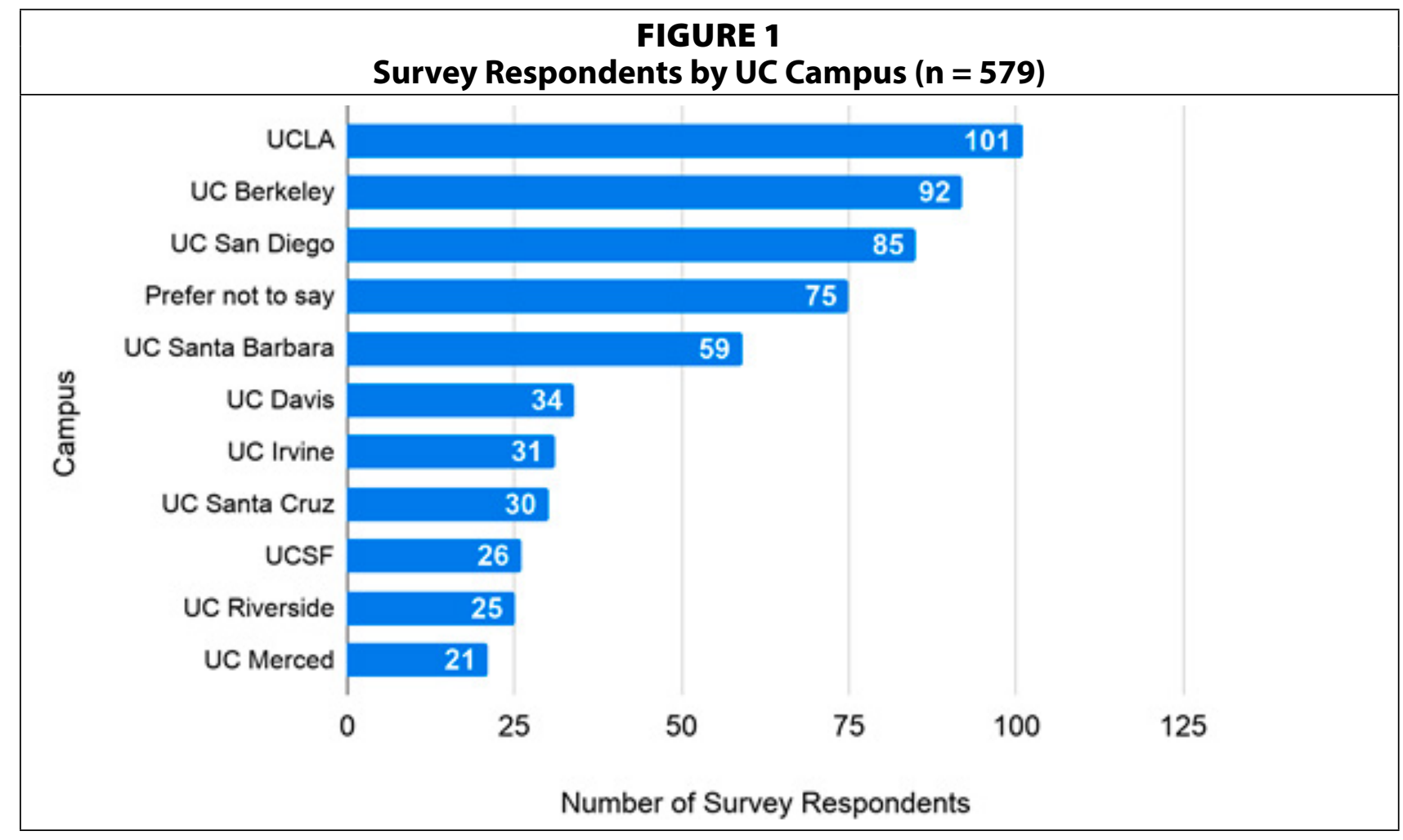




\section{Experiences and Behaviors}

"The behaviors happen while at service desks, when you are exposed to the public and feel like you cannot leave your post."

Fifty-four percent of UC Libraries employees $(n=313)$ who responded to our survey had experienced and/or observed sexual harassment at work. Of these, 63 percent were women, 20 percent were men, 15 percent preferred not to list their gender, and 2 percent represented an additional gender.

Every behavior we asked about in our survey was experienced and observed by library employees, with 313 respondents providing 1,016 responses (more than one behavior could be selected by each participant). Behaviors ranged from 9 to 225 individual responses, with 3 percent of participants having experienced and/or observed physical violence and 50 percent of respondents having experienced and/or observed gender harassment, defined as frequently treating you differently because of your gender. We asked participants how they experienced harassment: by experiencing it themselves, observing it directed at others, or both. Gender harassment and commenting on appearance were experienced much more than they were observed directed at others, while making sexual comments, staring or leering, and spreading sexual rumors were observed more than they were experienced (see figure 2).

Of the 313 participants who reported experiencing sexual harassment, more observed it directed at others than experiencing it themselves (see figure 3). Men were far more likely to observe harassment than experience it, while women experienced it more than they observed it.

\begin{tabular}{|r|r|r|r|}
\hline Sexual Harassment Behaviors Grouped by Experience Only, Observation Only, or Both \\
Experience and Observation
\end{tabular}




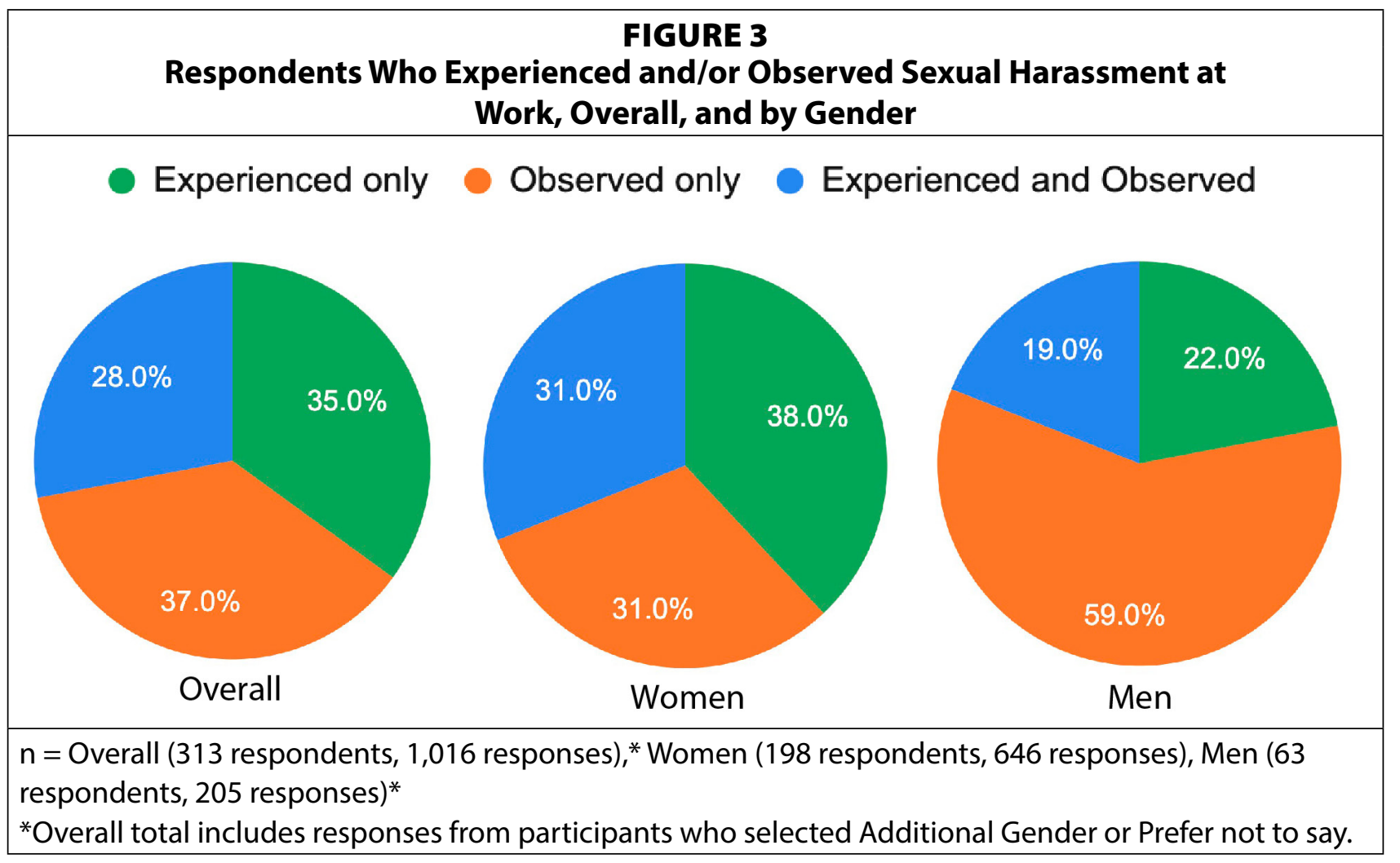

Figure 4 demonstrates the range of sexual harassment behaviors experienced and/or observed by UC Libraries employees by gender. Overall, there were no behaviors for which women experienced sexual harassment less than men. For each behavior, men observed harassment more than they experienced or experienced and observed it, and the reverse was true for women.

The free-text responses to the question demonstrated the variety of ways these types of behaviors can be exhibited and interpreted:

"I have experienced frequent comments on my appearance and unwanted hugs by UC Library staff. These actions were not at all malicious in nature but for me they went beyond professional boundaries and made me uncomfortable."

"Once a librarian had [sexually explicit material] on the shared computer at the front, [sic] circulation desk. He not only watched it, he insisted that I watch it, and anyone who walked by, including student employees, he did the same by pressuring all of [sic] to see it."

"Have been told repeatedly by co-workers and supervisors that the choice to be a mom and work part time during their childhood is damaging to my career and I should rethink."

"I was intimidated and assaulted by a patron."

Three-hundred thirteen participants representing 651 total responses (more than one option could be selected) demonstrated the range of exhibitors of sexual harassment of UC Libraries employees. The most common exhibitors were other UC Libraries staff, with 63 


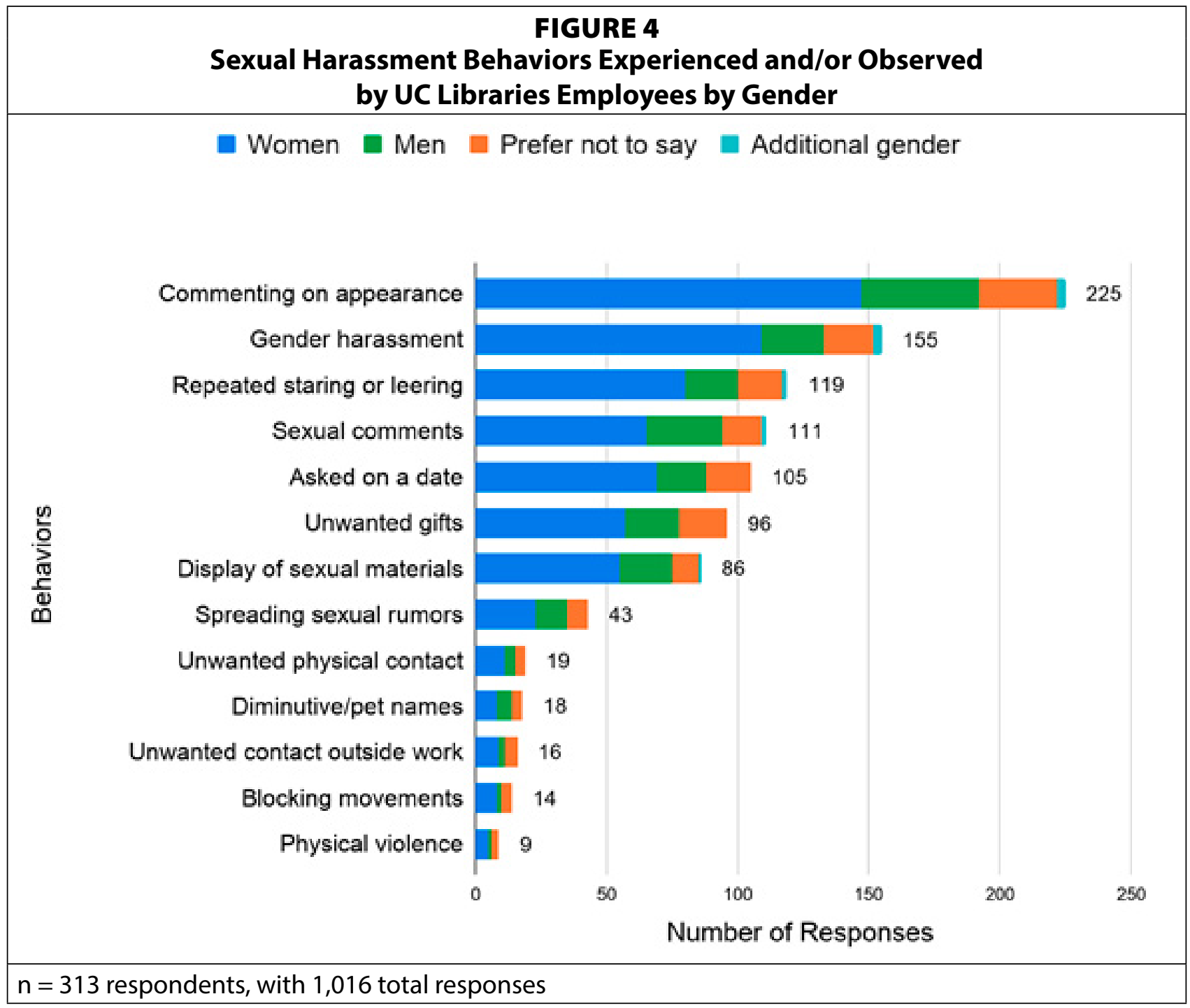

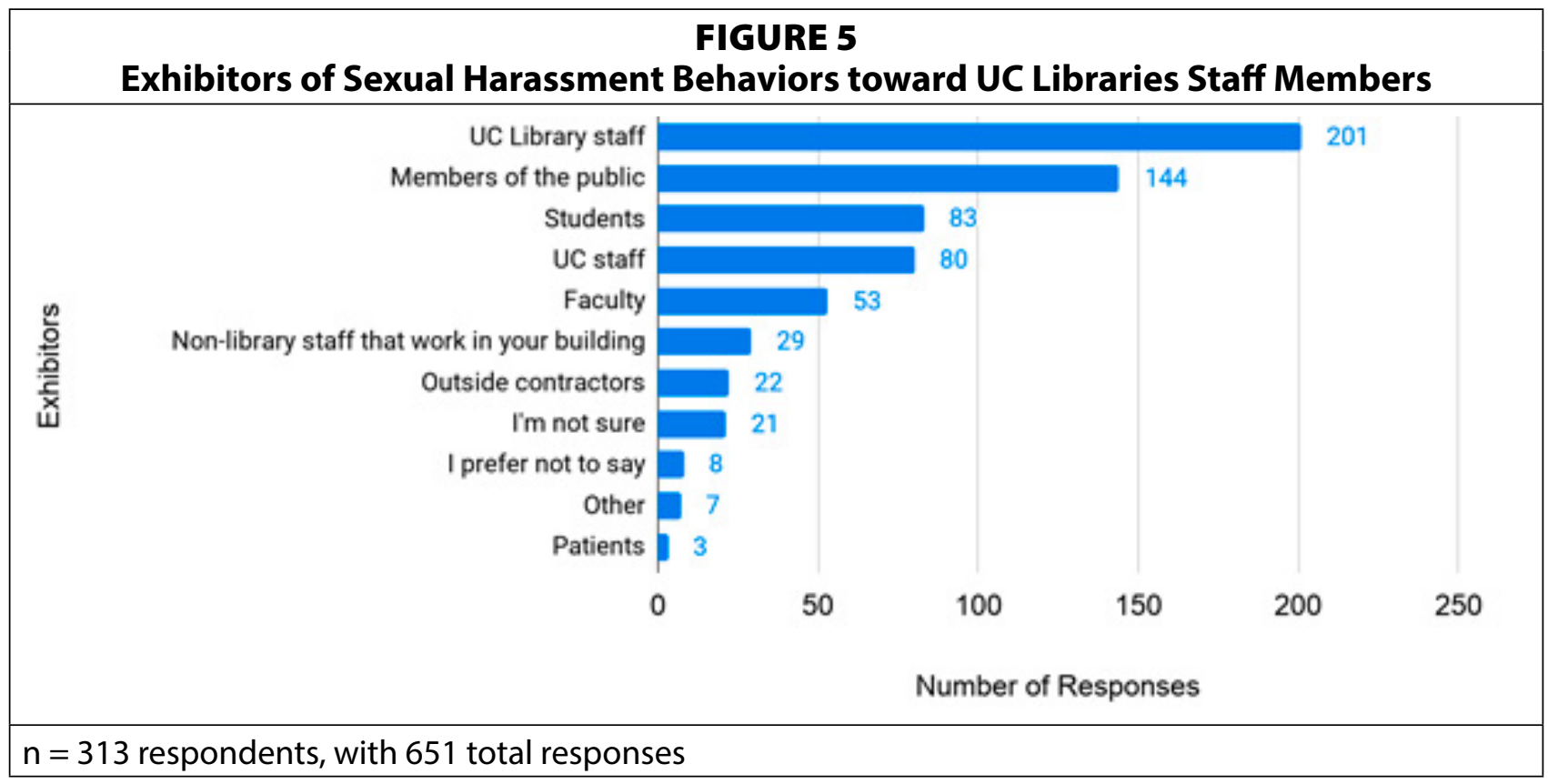


percent of respondents selecting this category, followed by members of the public (45\%) and students (26\%) (see figure 5). Corresponding with the overall results, women experienced sexual harassment from all exhibitors more than men. For example, of the participants who experienced and/or observed sexual harassment from UC Libraries staff, 61 percent were women and 20 percent were men; this trend was reflected for all categories of exhibitors.

\section{Reporting}

"A colleague had a donor make inappropriate comments at a fundraising event. The colleague told her supervisor and the response was 'leave well enough alone' due to the donor's importance to the library."

Seventy-four percent of respondents who experienced sexual harassment at work chose not to report or disclose their experiences, including 73 percent of women and 77 percent of men. Reasons why participants decided not to report or disclose their experiences or observances included feeling that the behaviors were not significant enough to report, believing that their experiences would not be taken seriously, fear of retaliation, and not knowing exactly where to report (see figure 6). The free-text responses demonstrated the variety of reasons why people chose not to report or disclose their experiences, as well as varying beliefs and attitudes about sexual harassment:

"The person worked here for a very long time and behavior was known and ignored."

"Previous reports by other people resulted in punishment of the victim."

"I was embarrassed and didn't want to make a fuss."

"If I were to report every time that I felt unfairly treated ... I'd spend more time reporting than working."

"The source of comments was an AUL [Associate University Librarian]."

Lack of knowledge of campus resources was a common theme for not reporting or disclosing sexual harassment. While 56 percent of respondents were aware of their Title IX office as a campus resource, far fewer were aware of other avenues to receive guidance, such as the UC's confidential CARE offices (18\%) or the Ombuds office $(14 \%)$.

Among respondents who did report or disclose their sexual harassment experiences, 93 percent did so out of a feeling of responsibility. Some cited that they were mandatory reporters, as one respondent noted, "I am a supervisor: I was required by law." Others noted a general concern for the safety of themselves and others in their libraries, such as, "I wanted to ensure this patron was not allowed in the library to repeat this behavior with other people." Among participants who did report or disclose sexual harassment, 32 percent responded that it was not clear where to do this, and 55 percent of people reported or disclosed to more than one place. Overall, most respondents who reported or disclosed sexual harassment did so to a supervisor (51\%), a colleague (47\%), or to Human Resources (33\%) (see figure 7). 


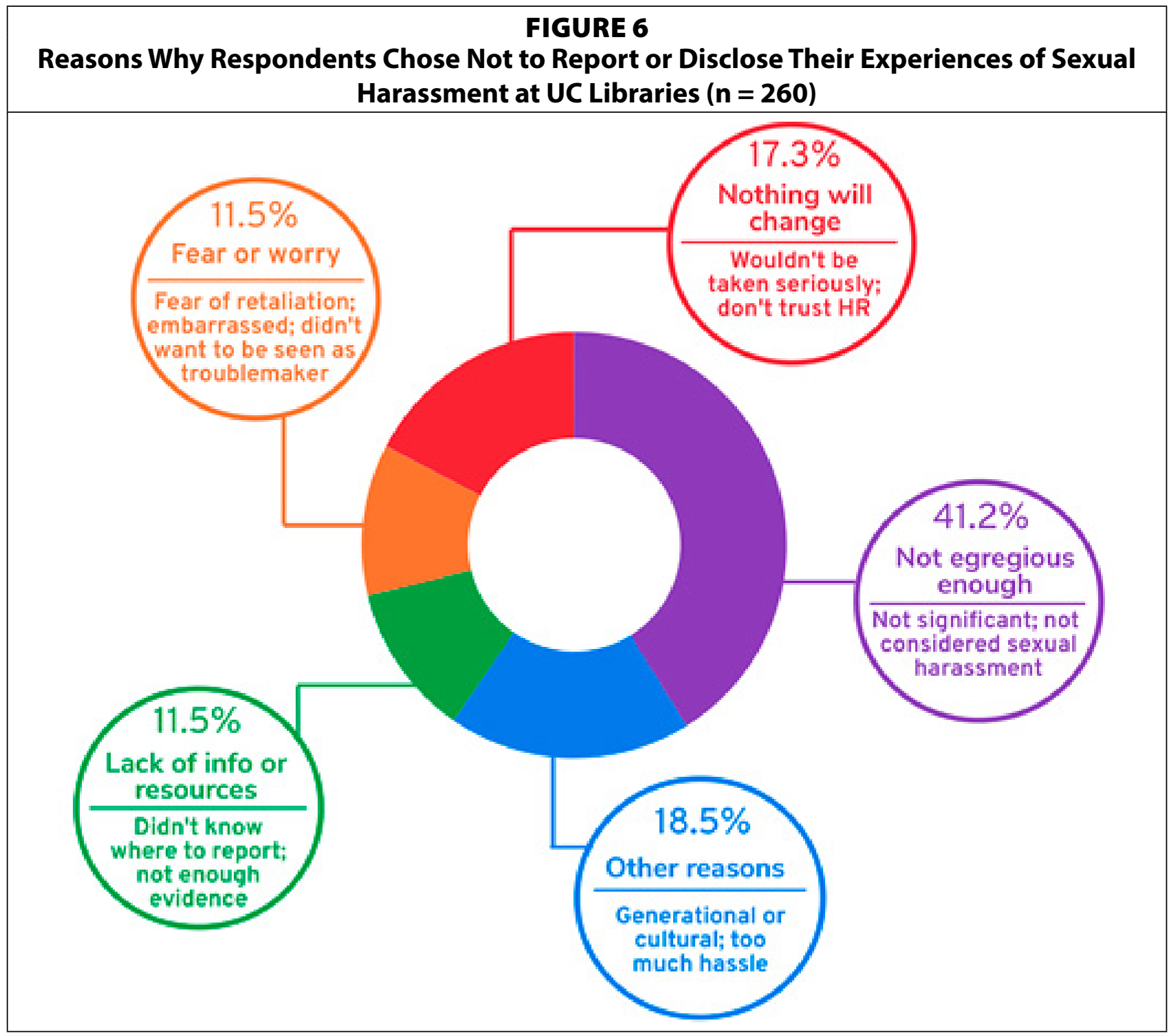

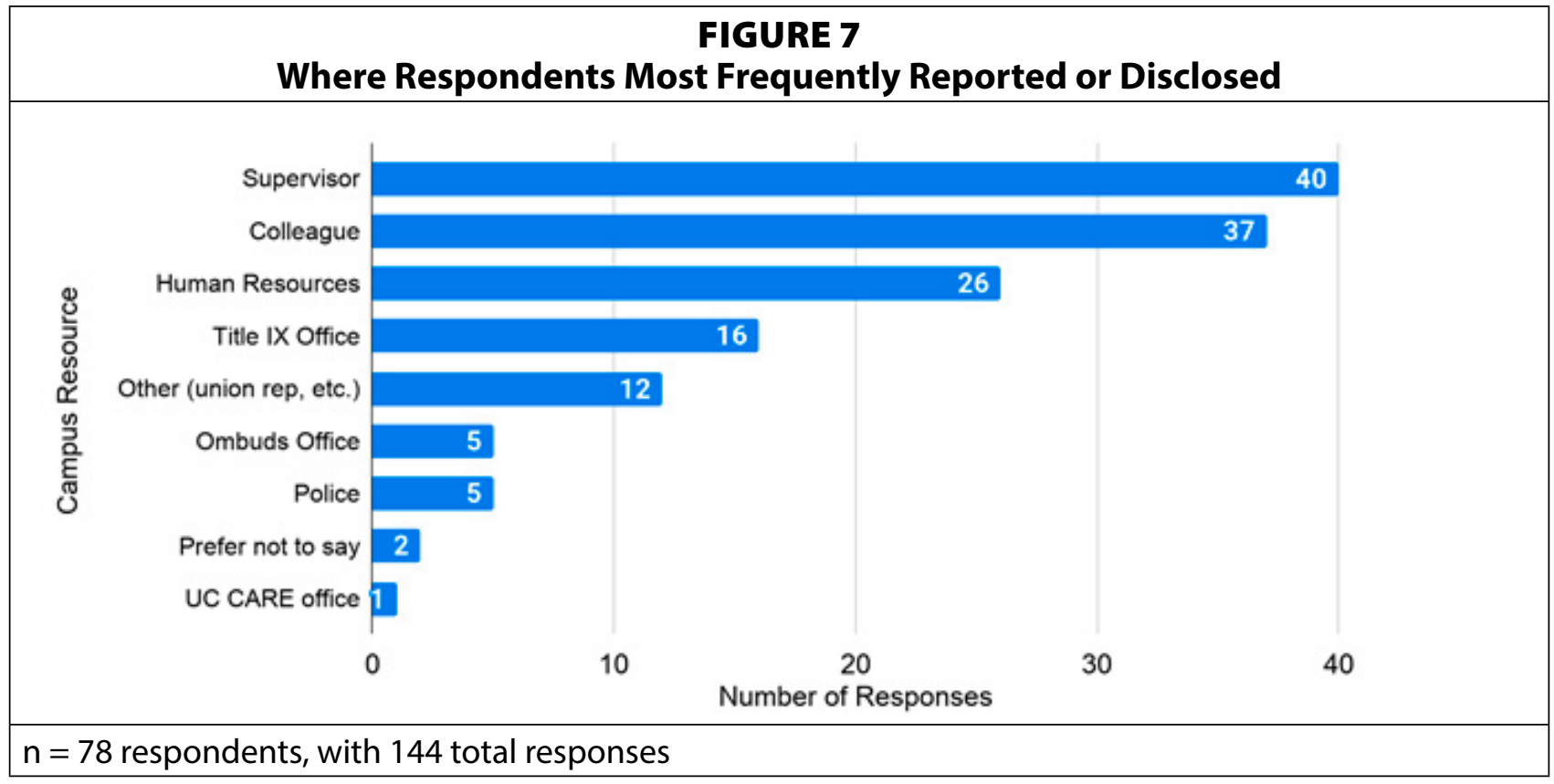


When asked if experiences of sexual harassment had impacted their work, the majority of participants said it had not (57\%), while 19 percent said it had affected their work, 22 percent were not sure, and 2 percent preferred not to say. Among respondents who believed these experiences had affected their work, 67 percent identified as women.

\section{Institutional Betrayal and Importance of Addressing Sexual Harassment}

"A grad student was coming to the library to stare at women. The women would complain, often in tears."

To get a sense of the relevance of this issue, we asked participants how important they considered addressing sexual harassment to be to themselves, to their UC campus library administration, and to their UC campus administration. While 66 percent of respondents felt that addressing sexual harassment at UC Libraries was very important to them, only 45 percent felt that their UC campus administration considered this issue to be very important, and fewer (43\%) felt that their own campus library administration considered this issue very important. Overall, very few respondents (1\%) felt that addressing sexual harassment was unimportant, 3 percent felt that their UC campus administration considered it unimportant, and a larger percentage $(5 \%)$ believed that their UC campus library administration considered it unimportant.

A set of our survey questions measured how respondents felt their UC campus administration and/or their library administration supported them with regard to their experience of sexual harassment. These questions only appeared for respondents who reported or disclosed to their Title IX office or supervisor (49 eligible, between 36 and 42 completed each question) and were measured using the 26-question Institutional Betrayal and Support Questionnaire described previously. ${ }^{16}$ We divided the questionnaire into three sections (Support, Betrayal, and Discrimination) and averaged the responses for each section to report overall scores and trends. Figure 8 lists examples of questions that were identified by participants as areas where the institution was not supportive; for the first six example questions under Support and Betrayal sections in figure 8, participants experienced betrayal more than support. For example, for the question "Did the institution allow you to have a say in how your report was handled?" 41 percent of respondents said no, 36 percent said yes, and 23 percent said n/a. While the majority of participants who reported or disclosed sexual harassment experiences felt supported by the institution (45\%,52\%, and 65\% for Support, Betrayal, and Discrimination sections, respectively), some felt unsupported, betrayed, or discriminated against during this process $(27 \%, 27 \%, 8 \%)$.

\section{Individual Campus Results}

"I'm not comfortable doing so because I'm concerned that, even if I omit names, describing some of my experiences would make it possible for the people who have performed these acts to identify me."

Overall, 13 percent of survey participants preferred not to state their campus, and 15 percent of people who experienced and/or observed harassment preferred not to do so. Among partici- 


\section{FIGURE 8}

\section{How Respondents Felt Supported, Betrayed, or Discriminated Against by Their Institution}

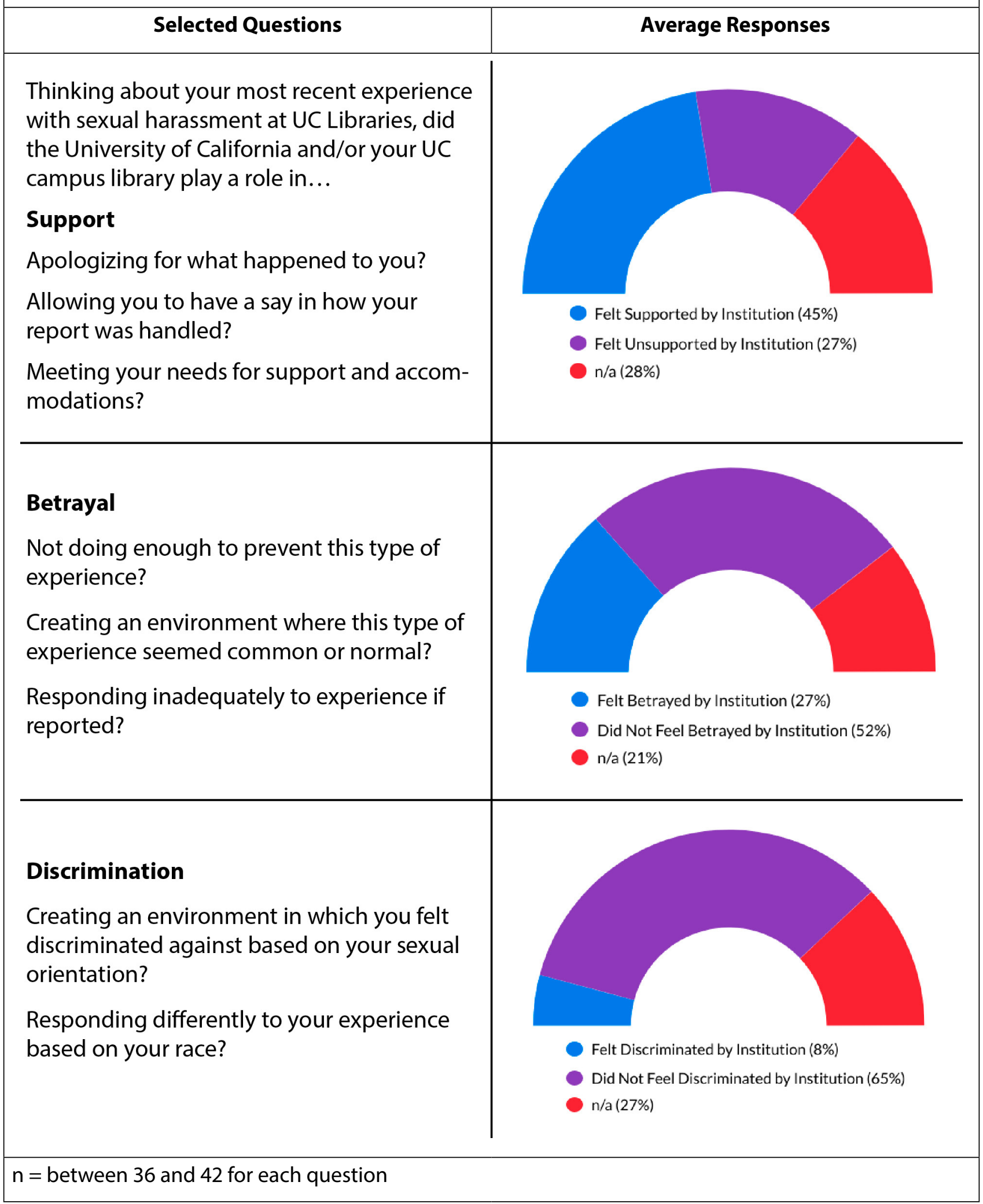


pants who selected their campus, results for each of the $10 \mathrm{UC}$ campuses reflected the overall results, with individual campus comparisons showing minimal differences across variables. We performed descriptive statistics only; significance testing was not conducted, as our goal was not to compare campuses but to provide customized results to each campus for their own information. Overall, campus response rates ranged from 22 (UCLA) to 68 percent (UC Merced), with a range of 39 (UC Irvine) to 69 percent (UC Berkeley) of respondents experiencing and/or observing sexual harassment, reflecting the overall rate of 54 percent. Behaviors were similar across campuses, with comments on appearance listed as the first or second most common behavior at 10 campuses and gender harassment at eight campuses. At eight campuses, UC Library staff were the most frequently listed exhibitor of sexual harassment, followed by members of the public. Among respondents who experienced and/or observed sexual harassment, between 11 (UC Merced) and 33 percent (UCLA \& UC Santa Cruz) reported or disclosed, most often to supervisors, Title IX offices, and Human Resources. Knowledge of the confidential CARE office as a resource varied by campus, ranging from 1 percent of participants (UC Irvine and UC Santa Cruz) to 23 percent (UC Berkeley). Additional details about specific campus results are listed in table 1.

\begin{tabular}{|l|c|c|c|}
\hline \multicolumn{4}{|c|}{ Detailed Results by Campus } \\
\hline & $\begin{array}{l}\text { Response } \\
\text { Rate }\end{array}$ & $\begin{array}{l}\text { Percent Who Have } \\
\text { Experienced/Observed } \\
\text { Sexual Harassment }\end{array}$ & $\begin{array}{l}\text { Percent Who Have } \\
\text { Reported Their } \\
\text { Experiences }\end{array}$ \\
\hline UC Berkeley & $26 \%$ & $69 \%$ & $23 \%$ \\
\hline UC Davis & $27 \%$ & $44 \%$ & $27 \%$ \\
\hline UC Irvine & $20 \%$ & $39 \%$ & $25 \%$ \\
\hline UCLA & $22 \%$ & $47 \%$ & $33 \%$ \\
\hline UC Merced & $68 \%$ & $43 \%$ & $11 \%$ \\
\hline UC Riverside & $30 \%$ & $56 \%$ & $31 \%$ \\
\hline UC San Diego & $36 \%$ & $47 \%$ & $24 \%$ \\
\hline UC San Francisco & $42 \%$ & $65 \%$ & $26 \%$ \\
\hline UC Santa Barbara & $45 \%$ & $56 \%$ & $21 \%$ \\
\hline UC Santa Cruz & $45 \%$ & $50 \%$ & $33 \%$ \\
\hline Preferred not to state campus & N/A & $63 \%$ & $32 \%$ \\
\hline
\end{tabular}

\section{Intersectionality}

"I am not sure if it is because I am a woman/female, but I get asked to do the cleaning up of space, or tidying up of space, more so than my male counterpart. I get that I am good at it, but it seemed like my boss picked on the women to do that sort of manual labor. Didn't feel right."

Fifty-nine participants wrote in responses that reflected on the parts of their identity (such as gender, race, sexual orientation, socioeconomic class) they felt affected their experiences of sexual harassment at UC Libraries. Because of the individual and heterogeneous nature of these experi- 
ences, we found it difficult to draw conclusions from this data; however, the number of responses suggests that this is an area where further study is warranted. Although we were unable to draw conclusions from these disparate findings, we have categorized responses by major themes, which indicate that many respondents found these aspects of their identities to be important and suggest that gender and race, in particular, are important in this discussion (see figure 9).

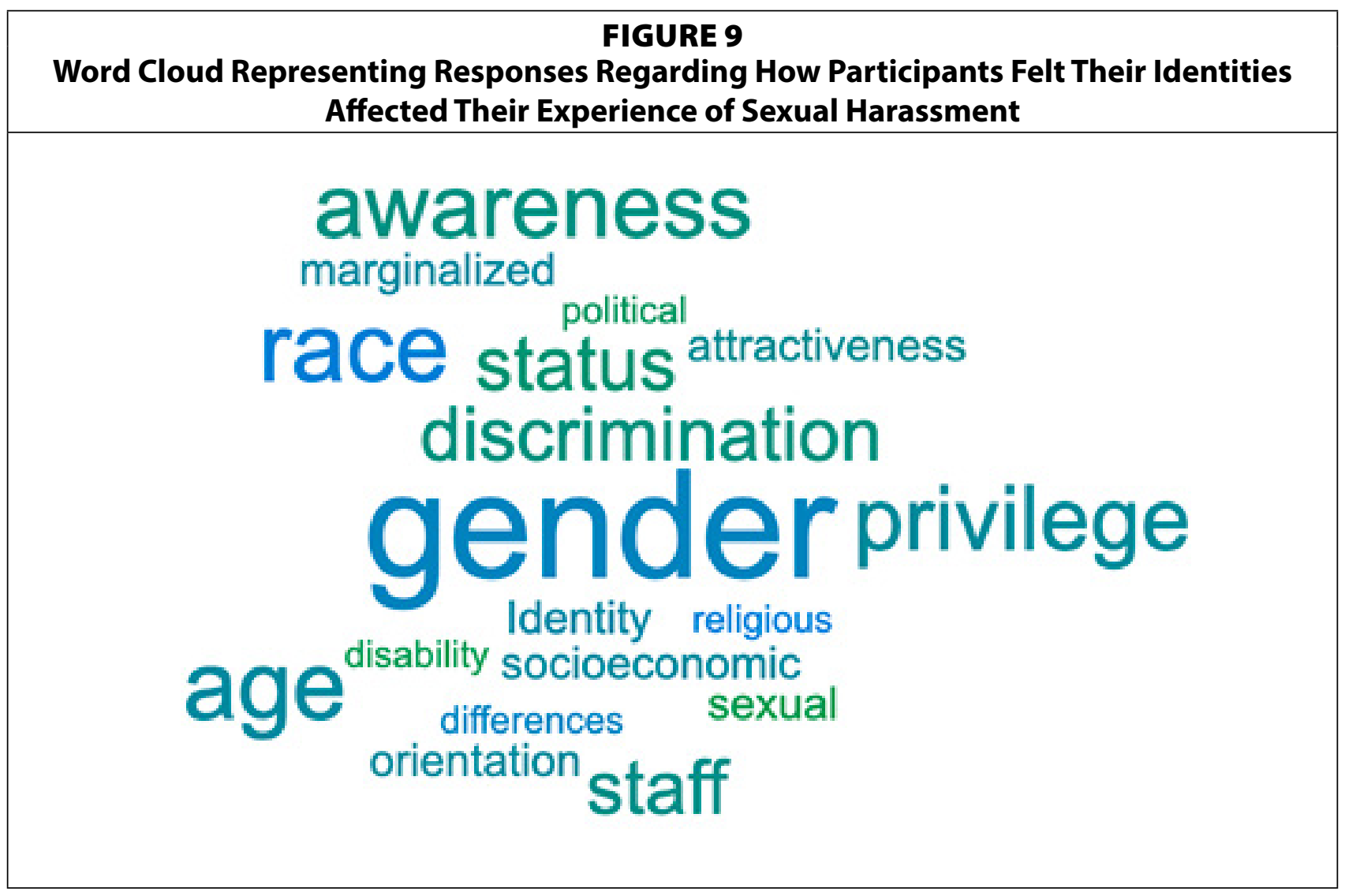

\section{Recommendations}

"Administration does not seem to treat complaints from staff as seriously as they do from students."

We received 224 responses to this question: "What kinds of steps or actions would you like to see from UC Libraries or your campus library in addressing sexual harassment?" Although all University of California employees are required to complete mandatory sexual harassment training every two years, ${ }^{17}$ the most frequent recommendation from participants was around training and education, with specific requests that it be mandatory, in-person, and focused on bystander interventions, role-playing, and resources for reporting. Four additional themes emerged as recommendations: workplace culture change; consequences for harassers; support from leadership, including clear communication and regular statements on this issue; and clear, documented reporting guidelines and processes. Responses to this question highlighted disparities in experiences among UC Libraries staff members: while 20 percent of respondents believed additional actions were not needed, 13 percent reported that the current administration is not supportive around this issue. 


\section{Discussion}

"When I told my supervisor the response was 'Oh, that's just how they act; no need to worry' or 'Okay, I will pass on the info' with no follow-up."

Sexual harassment clearly exists at UC campuses, and there is space for work to be done to combat it. Happily, at most UC campuses, there is a desire among staff and leadership to take action going forward. In the time since our study was completed, we visited 8 of 10 UC campuses to share overall and campus-specific results and facilitated discussions about our study's recommendations. Two of the 10 campuses (UC Davis and UC Riverside) responded that they were not interested in a presentation by our study team and their campus CARE director to discuss campus-specific results. All UC Libraries employees were also invited to a webinar to discuss study findings. As a result of our work, several UC campus libraries have held brainstorming sessions on this topic, incorporated CARE representatives into training efforts, and folded efforts into larger diversity-focused initiatives. It has been heartening to see this progress being made in so many different places, even though there is clearly much work to be done. During campus visits, we invited CARE and Title IX representatives to copresent our sessions to provide additional information, answer questions from library staff in the context of our study results, and facilitate a connection between these offices and each campus library. Although most survey participants were aware of their Title IX office as a resource, an overwhelming majority of library employees who completed the survey $(99 \%$ on some campuses) were not aware of CARE's confidential support services for sexual harassment. We recommend partnering with both of these groups (or their equivalents) to raise awareness of these services and use the expertise of those familiar with the campus landscape, policies, and resources in this area.

One question raised by our study is the role of training in preventing and addressing sexual harassment at work. Interestingly, the most frequent recommendation from survey participants was training or education of some kind. With more university systems requiring regular training on this issue, it is worth investigating how training effectiveness is measured and tracked and how improvements might be made to current training efforts. The University of California requires all employees to participate in mandatory sexual harassment training every two years, ${ }^{18}$ yet sexual harassment is still happening. Perhaps implementing the recommendations of survey participants, including an in-person training that includes role-playing, bystander intervention scenarios, situations specific to libraries, and a focus on reporting guidelines would allow employees to feel more empowered; this remains to be seen.

Our study findings may also assist in developing training informed by the real-life experiences of library staff that meets their self-identified needs. As UC Library staff were the most frequent exhibitors of sexual harassment behaviors, training could first focus on this population. Additionally, we found that gender harassment and commenting on appearance were the most frequent types of behaviors experienced and/or observed by study participants, which is consistent with existing research on sexual harassment experiences in academia. ${ }^{19}$ Importantly, these two behaviors were experienced more often than observed, while making sexual comments, staring or leering, and spreading sexual rumors were observed more often than experienced. Thus, there may be opportunities for bystander training for certain types of behaviors, combined with education about what sexual and gender harassment can look like 
to address various behaviors according to how they're actually experienced and observed by library staff. In addition to the fact that women experience harassment more than men, our findings showed that UC Libraries employees have different experiences of sexual harassment based on their individual identities and how these intersect. There is an opportunity to include these considerations in designing training efforts that highlight differences in the ways that people experience sexual harassment. Finally, our findings about the places participants report or disclose indicate a need for education about reporting resources, particularly regarding the fact that more than 30 percent of people did not know where to report on their campus, and 82 percent (between $77 \%$ and $99 \%$ on individual campuses) were not aware of CARE's confidential support services. Supervisors, colleagues, and Human Resources, the top three places that participants identified as reporting or disclosing to, are all located in the library, indicating a need for library staff training that highlights available resources for employees interested in reporting or disclosing their experiences.

\section{Moving Forward}

"They weren't acts of sexual harassment. They were part of normal daily interaction. If we are always looking for something wrong, you will find everything offensive."

While most library employees were supportive of our study, we encountered pushback from some individuals and campus representatives. The fact that some respondents considered addressing sexual harassment to be unimportant and 5 percent believed their UC campus library considered this issue unimportant speaks to the different experiences and beliefs of library staff across the UC system. Similarly, the varied experiences of support, betrayal, and discrimination of those disclosing or reporting sexual harassment points to the fact that this process can be greatly improved. The 15 percent of participants who experienced and/or observed sexual harassment and did not feel comfortable listing their campus speaks to the complexity of this issue and the lack of support that some UC library employees have experienced. These findings, combined with the lack of interest of two UC campuses in a discussion of campus-specific results, suggests that there is still much work to be done to address sexual harassment at UC Libraries, and explicit support for this work needs to come from library leadership. The resistance to combating sexual harassment or even to discussing it strikes us as counterproductive, and we wonder about the viability of efforts to eliminate harassment without buy-in from important campus community members. As is reflected in the literature, our study found that experiences of sexual harassment can affect one's work, and a lack of support from institutions can cause disruptions to work and sustainment of an unsupportive culture. ${ }^{20}$ In an academic library setting, this can affect quality of services and team morale. Additionally, if sexual harassment occurs within a library space, it is quite possible that, because they work where an incident occurred, UC Library employees can be triggered during other face-to-face interactions. While the goal of this study was to create recommendations for UC Libraries, our experiences conducting this research is an indication that taking action based upon this study will likely vary by campus across the UC Libraries system.

During one campus presentation, a library staff member asked how to foster cultural change, and the response we gave was to increase trust and accountability. This exchange reflects participant recommendations for support from leadership, consequences for harass- 
ers, and clearer reporting guidelines, though trust and accountability may be hard to measure and harder to instill. In the case of our study, if a UC campus does not recognize the importance and impact of sexual harassment, it can be difficult to foster trust and accountability throughout the system, requiring these qualities to stem from leadership beyond the library organization. Working within the organizational culture of the larger campus community may reveal benefits - or pose increased bureaucratic challenges. In one recent example, federal rules released in May 2020 that narrow definitions of sexual misconduct and limit rights of Title IX offices to conduct certain investigations were opposed by University of California president Janet Napolitano, particularly for the possibility that they "could deter already reluctant and sometimes traumatized complainants from coming forward." ${ }^{21}$ Our study found that only 26 percent of participants who experienced sexual harassment reported their experiences; the further chilling effect of these new rules remains to be seen. Despite these external challenges, our study findings can provide a start for other libraries to think through the challenge of creating culture change within their environments, perhaps even starting with individual departments.

\section{Limitations}

"In a department which adheres to policy and has an Org Chart and clear approval routing, a co-worker said to me that our Director 'lets me do whatever I want' and suggested to others that I was having an affair w/ the Director."

Our study has several limitations. While we made an effort to raise awareness about our study through the use of an Advisory Team, response rates varied widely across campuses, indicating that some campuses may have been underrepresented in our overall results. Because this study deals with a topic that can elicit varied reactions from participants, including survivors, those who experienced sexual harassment may be underrepresented. Similarly, those who have never experienced or observed sexual harassment may not have participated in the study. We attempted to counter this selection bias by working with CARE representatives to consider survey language from a survivor standpoint and by specifically stating in our survey invitation that those who have not experienced sexual harassment were encouraged to participate. We did not survey the experiences of student workers in our study; this remains an understudied area, as library student workers are often in forward-facing roles requiring them to interact with users. We were unable to collect demographic information including race and sexual orientation in our survey; this prevented us from making meaningful conclusions about the experiences of library employees from marginalized groups. Our results indicate that intersectionality affects experiences of sexual harassment, and we believe that more research is needed on this topic to understand these experiences. We used descriptive statistics, rather than significance testing of relationships, so we are unable to compare the experiences of employees from different campuses. This decision was made because we wanted to share results with each campus, rather than turn our findings into a competition between campuses or highlight results from any single institution; we believe this would be unproductive in starting conversations about addressing sexual harassment at individual UC campus libraries and across the UC Libraries system as a whole. Finally, we did not use an existing survey instrument like the Sexual Experiences Questionnaire, ${ }^{22}$ and our results may not be generalizable to other academic librar- 
ies, as we focused solely on the University of California system. However, because we were conducting action research with the goal of creating change at our institution, we believe our decision to measure behaviors and exhibitors that reflected individual experiences can better inform training efforts and other changes going forward.

\section{Conclusion}

"The comments I received and have observed others receiving are seemingly innocuous. However, I think this is part of the issue that something that seems not harmful still can be harmful."

We measured sexual harassment experiences of 1,610 nonstudent employees at University of California Libraries, with 579 respondents (36\%) participating in our study. Sexual harassment is happening at our libraries in various forms, is exhibited most often by UC Libraries staff members, and is not often being reported. UC Libraries employees feel supported by their institutions overall, but some have experienced institutional betrayal during the reporting process, and less than half of participants think their library considers addressing sexual harassment to be a very important issue. Culture change, support from leadership, consequences for harassers, and clear reporting processes are recommended by library employees to address sexual harassment. While training and education efforts were the most frequent recommendation, it remains to be seen if additional training will address sexual harassment at UC Libraries and beyond.

\section{Acknowledgments}

We would like to thank our Advisory Team: Jacqueline Bates, Allison R. Benedetti, Nicole E. Brown, Christy Brown Anderson, Heather K. Hughes, Jennifer Lee, Sarah Lindsey, Shu Liu, Jessica Pierucci, Philippe Robles-Fradet, Monalisa Ruiz, and Lynda Tolly; without their guidance and support, this study would not have been started, let alone completed. CARE and Title IX staff across the UC system graciously shared their expertise and time with us: the efforts of Karla Aguilar, Yesenia Curiel, Alison Hanson, Jared Jorde, Sarah Meredith, Mandy Mount, Julia Pennick, Elizabeth Rogers, and Liat Wexler are particularly appreciated. Special thanks to Denise Caramagno, LMFT, UCSF CARE, for coordinating connections to CARE directors and advocates, and for her continued guidance throughout the study. Thanks to our colleagues who provided feedback on this manuscript: Sara Davidson Squibb, Annie Pho, Micquel Little, and Chris Shaffer. Thank you to members of CoUL and ASAG who supported our study and those who shared their critiques with us openly. Finally, thank you to the 2018 LAUC Executive Board who helped us begin our study by sending out a call for advisory team members. We've been humbled by the outpouring of support across the UC system at every stage of this project and would like to thank our colleagues continuing this important work going forward. 


\section{APPENDIX A Study protocol}

Title

Sexual harassment at University of California Libraries: understanding the experiences of library staff members

\section{Study team}

Study team: Jill Barr-Walker, UCSF (PI), Nisha Mody, UCLA, Elizabeth McMunn-Tetangco, UCM, Courtney Hoffner, UCLA

Advisory team: This will be made up of one representative from each UC campus library and will meet on a quarterly basis. The advisory team will provide feedback on our study aims, survey content, dissemination plans, and future actions. We will also solicit representation from CARE advocates across the UC system in our advisory team. The goal of creating the advisory team is to ensure that our study considers the unique needs of each UC campus library.

\section{Summary}

Sexual harassment of academic library staff members has not been comprehensively studied. A recent pilot study that surveyed UCSF Library staff about sexual harassment experiences found that $48 \%$ of respondents had experienced sexual harassment at work, involving a range of behaviors from a variety of people in the library. A summary of the results of that study can be found here.

The current study aims to expand that project and explore sexual harassment experiences of University of California Library employees across the 10 UC campuses. We will survey UC Library employees about their experiences with sexual harassment, including which types of behaviors have been experienced and from whom. Data will be collected via an anonymous survey emailed to all UC Library employees (around 1610 people). We will analyze the data using descriptive statistics and bivariate analyses comparing relationships between sexual harassment experiences and race, gender, UC campus, and other variables. We will present our findings, including campus-specific reports, to Library stakeholders including CoUL, LAUC, and all UC Library staff, along with recommendations for training and policy changes that are informed by the data. We will use our results to raise awareness about sexual harassment and begin conversations, both locally and system-wide, about how to address this issue across UC Libraries.

\section{Aim}

The aim of this study is to explore workplace sexual harassment experiences of University of California Library employees.

\section{Objectives}

- Measure sexual harassment experiences quantitatively, including types of behaviors experienced and user categories of those who exhibit behaviors, and qualitatively 
- Measure opinions of library staff regarding institutional support around sexual harassment

- Produce policy and training recommendations for University of California Libraries based on this data

\section{Research questions}

- What types of sexual harassment behaviors have UC library staff members experienced?

- Who exhibits sexual harassment behaviors to UC library staff members?

- Are there differences in sexual harassment experiences (either by type or frequency) across UC campuses?

- What associations exist between sexual harassment experiences and race, gender, and/or job type?

- How do UC Library employees view their institutions' actions around sexual harassment?

\section{Literature review}

No large-scale study of sexual harassment in academic libraries has been conducted. A recent Book Riot article collected data from library staff members in an informal online survey and identified sexual harassment as a problem across library types, highlighting a range of sexual harassment behaviors including verbal and physical harassment. Very recent work has been done on this topic, including several presentations and articles by librarians at Waukegan Public Library in the past year $[1,2]$ and an ongoing survey about sexual harassment in academic libraries being conducted by staff at Radford University and William \& Mary University. Our study differs from these projects as it will examine the experiences of library employees at one academic institution, the University of California, in order to determine the scope of this issue within our library system and recommend actionable goals to address it. We are also interested in examining the experiences of all library staff, not just academic librarians.

Our PI, Jill Barr-Walker, previously collected data about sexual harassment experiences at UCSF Library (February 2018) which revealed the existence of this issue at one UC library. A summary of the results of that pilot study can be found here. Our goal is to measure similar experiences at all UC libraries with the hope of starting conversations about how to address sexual harassment of UC library staff.

\section{Study population}

We will survey all University of California library staff members at 10 UC campuses. The total study population is estimated at 1610 . 


\section{Sampling design}

We will conduct a census of all UC Library employees.

\section{Data collection instruments}

Participants will complete an anonymous online survey that asks for information about the following: 1) which sexual harassment behaviors they have experienced at their UC Library, 2) who they have experienced sexual harassment behaviors from (this will be measured in user categories, e.g. UC staff, library staff, members of the public), 3) how they feel their UC Library and the University of California has supported them with regard to their experience of sexual harassment (this will be measured using the Institutional Betrayal Questionnaire), and 4) how UC Libraries can address sexual harassment in the future. Sexual harassment behaviors listed in the survey will be selected through an iterative process involving feedback from our Advisory team, CARE advocates, and use of existing scales. Demographic information, including gender, race, UC campus, and job type will also be collected.

All survey questions are optional and participants are invited to skip questions or stop the survey at any time if they do not feel comfortable. The responses will be anonymous; some demographic categories (e.g. race/ethnicity) are not being collected to keep participant identities confidential. Because we believe it is important to understand the experiences of our library employees of color and identify potential racial disparities, we will ask participants to select their racial identities from broad categories (i.e. white, person of color) which will protect anonymity. Participants will not receive incentives for participating. The survey will be hosted by REDCap, a secure, web-based application designed to support data capture for research studies.

\section{Data analysis techniques}

We will analyze the data, using mostly descriptive statistics. We may use bivariate analyses to test relationships, such as experience of sexual harassment behaviors and a variety of variables including gender, race, UC campus location, and job type. All analyses will be conducted using Microsoft Excel and Stata.

\section{Dissemination of results}

We will share our data and findings with all UC Library employees after removing any potentially identifying information. We will present our results at each UC campus using customized reports. We also hope to share our findings at academic conferences and in journals.

\section{Next steps / future plans}

We will encourage the creation of a CKG or task force that will address the results of the study and recommend actionable goals to eliminate sexual harassment within the UC Library system. 
These goals may be created and executed by each campus or across the UC Library system, depending on relevance and need.

\section{Project schedule}

\begin{tabular}{|c|c|c|c|c|c|c|c|c|c|c|c|c|c|}
\hline Task & $\begin{array}{l}\text { May } \\
' 18\end{array}$ & $\begin{array}{l}\text { Jun } \\
\text { '18 }\end{array}$ & $\begin{array}{l}\text { Jul } \\
' 18\end{array}$ & $\begin{array}{l}\text { Aug } \\
' 18\end{array}$ & $\begin{array}{l}\text { Sept } \\
' 18\end{array}$ & $\begin{array}{l}\text { Oct } \\
118\end{array}$ & $\begin{array}{l}\text { Nov } \\
' 18\end{array}$ & $\begin{array}{l}\text { Dec } \\
' 18\end{array}$ & $\begin{array}{l}\text { Jan } \\
' 19\end{array}$ & $\begin{array}{l}\text { Feb } \\
' 19\end{array}$ & $\begin{array}{l}\text { Mar } \\
19\end{array}$ & $\begin{array}{l}\text { Apr } \\
' 19\end{array}$ & $\begin{array}{l}\text { May } \\
' 19\end{array}$ \\
\hline $\begin{array}{l}\text { Obtain support } \\
\text { from LAUC \& } \\
\text { CoUL }\end{array}$ & $x$ & $x$ & & & & & & & & & & & \\
\hline $\begin{array}{l}\text { Create study team } \\
\& \text { advisory team }\end{array}$ & $x$ & $x$ & & & & & & & & & & & \\
\hline Create survey & $x$ & $x$ & $x$ & $x$ & $x$ & & & & & & & & \\
\hline IRB approval & & & $x$ & $x$ & $x$ & & & & & & & & \\
\hline Collect survey data & & & & & & & $x$ & $x$ & & & & & \\
\hline $\begin{array}{l}\text { Apply for LAUC } \\
\text { grant }\end{array}$ & & & & & & $x$ & & & & & & & \\
\hline Analyze data & & & & & & & & $x$ & $x$ & $x$ & & & \\
\hline $\begin{array}{l}\text { Share results at } 10 \\
\text { UC campuses }\end{array}$ & & & & & & & & & & & $x$ & $x$ & $x$ \\
\hline $\begin{array}{l}\text { Begin discussions } \\
\text { about CKG or task } \\
\text { forces for next } \\
\text { steps }\end{array}$ & & & & & & & & & & & $x$ & $x$ & $x$ \\
\hline
\end{tabular}

\section{References}

Civitello, A. (2017). \#MeToo: Confronting sexual harassment in the public library. Public Libraries, 56(6), 9-10.

Civitello, A. \& McLain, K. (2017). It's not just part of the job: Speaking out against sexual harassment. ILA Reporter, December. 


\section{APPENDIX B. Survey instrument}

\section{Confidential \\ Sexual harassment at University of California Libraries: understanding the experiences of library staff members}

We are inviting you to take part in a research study conducted by Jill Barr-Walker and colleagues across UC Libraries (UCSF IRB\#18-25552). If you choose to participate in the study, you will complete a survey to help us learn more about sexual harassment at University of California Libraries. The survey will take approximately 15 minutes to complete.

The survey's questions are about your experiences with sexual harassment in the University of California Libraries. Your participation is optional. If you are willing to take part, you will help us understand how sexual harassment is experienced by UC Libraries employees.

We understand that some questions may be difficult to answer or remind you of negative or traumatic experiences; if you are not comfortable responding to any of the questions, please feel free to skip questions and/or stop the survey at any time. If you would like to speak with someone about your experiences, you can contact a CARE advocate for free and confidential support. The CARE Advocate's contact information for your campus can be found here: http://tiny.ucsf.edu/pmDKOm.

This survey is anonymous, and there is no way for your responses to be linked back to you. Because the survey is anonymous, we will not make any mandated reports based on information that you share. To ensure that we follow the University of California mandated reporting policy, please do not share any potentially identifiable information (e.g. your name, your department, or specific details about people involved in any unreported incidents).

Survey results, including quotes from text provided in write-in sections, may be shared with UC Libraries staff, other members of the UC community, and within academic publications and presentations. This data will be shared in order to raise awareness about sexual harassment at UC Libraries and within the library workforce on a local and national level. We hope to use this data to inform change efforts within our organization and potentially the library profession as a whole.

Questions? Please contact jill Barr-Walker at jill.barr-walker@ucsf.edu. If you have questions or concerns about your rights as a research participant, you can call the UCSF Institutional Review Board at 415-476-1814.

To participate in this study, please start the survey on the next page.

As a reminder, this survey is anonymous; please do not share any potentially identifiable information. If you aren't comfortable responding to any part of the survey, feel free to skip questions or stop the survey at any time.

For the purposes of this study, we define "sexual harassment" as unwanted behaviors that can make you uncomfortable at work and are of a sexual nature and/or based on your gender.

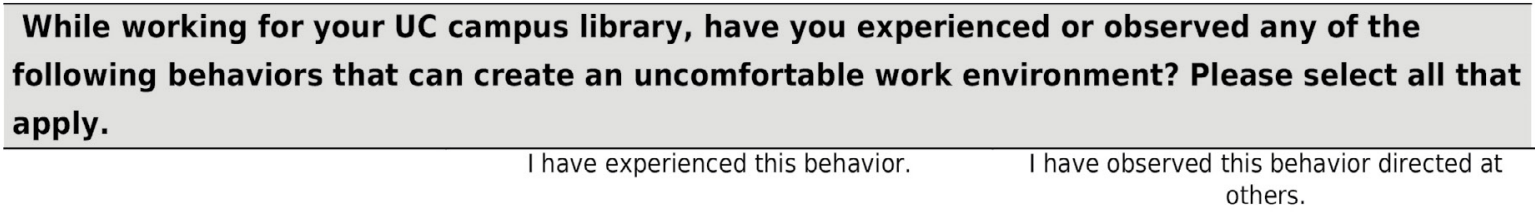

Asking you on a date

Bringing you unwanted gifts

Commenting on your

appearance 


\section{Confidential}

Displays of sexually suggestive materials (including on computer screens or phones)

Frequently treating you differently because of your gender (including favoring, slighting or ignoring, or making offensive remarks based on your gender)

Making sexual comments (including jokes, gestures, slurs, or derogatory comments)

Repeated staring or leering Spreading sexual rumors Unwanted contact outside of work (e.g. friending you on Facebook or calling your cell phone)

Use of diminutive or pet names

Blocking your movements

Unwanted physical contact of any kind

Physical violence of any kind, including but not limited to hitting, pushing, restraining or unwanted sexual contact

Other

Please specify.

I have not experienced or observed any of these behaviors while working for my UC campus library.

If you are comfortable doing so, feel free to share more information about your experiences with the behaviors listed above. Please do not include names or any other identifying characteristics to ensure compliance with mandated reporting laws. Some text from responses may be shared in reports generated from the survey results. 


\section{Confidential}

Who has exhibited the above behaviors? Please select all that apply.

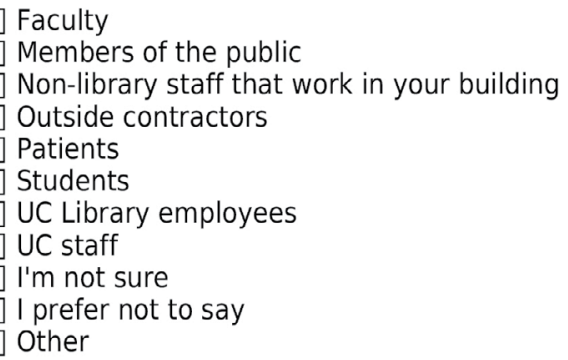

Please specify.

\section{Confidential}

Which resources do you know of on your campus where you could make a report of sexual harassment?

\begin{tabular}{|c|c|}
\hline $\begin{array}{l}\text { Did you report or disclose your experiences of (or } \\
\text { experiences observing) sexual harassment at your UC } \\
\text { campus library to anyone? }\end{array}$ & $\begin{array}{l}\bigcirc \text { Yes } \\
\text { No } \\
\text { I prefer not to say }\end{array}$ \\
\hline $\begin{array}{l}\text { Who did you report or disclose to? Select all that } \\
\text { apply. }\end{array}$ & $\begin{array}{l}\square \text { Colleague } \\
\square \text { Confidential CARE advocate } \\
\square \text { Human Resources } \\
\square \text { Office of the Ombuds } \\
\square \text { Police } \\
\square \text { Supervisor } \\
\square \text { Title IX office } \\
\square \text { Other } \\
\square \text { I prefer not to say. }\end{array}$ \\
\hline
\end{tabular}

Please specify.

Was it clear where to report?

$\bigcirc$ Yes

No

I prefer not to say

What factors influenced your decision to

report/disclose or not report/disclose?

\section{Confidential}

The following questions are from an existing scale used to measure institutional support around sexual harassment. If you are not comfortable responding to any of the questions, please feel free to skip questions and/or stop the survey at any time. If you would like to speak with someone about your experiences, you can contact a Campus Advocacy Resources \& Education (CARE) advocate for free and confidential support. The CARE Advocate's contact information for your campus can be found here: http://tiny.ucsf.edu/pmDKOm. 
Thinking about your most recent experience with sexual harassment at UC Libraries, did the University of California and/or your UC campus library play a role in....

Actively supporting you with
either formal or informal
resources (e.g., counseling,
academic services, meetings or
phone calls)?

Apologizing for what happened to you?

Believing your report?

Allowing you to have a say in how your report was handled?

\section{Yes}

$\mathrm{O}$

\section{No}

$\mathrm{O}$
N/A

$\mathrm{O}$

Ensuring you were treated as an important member of the institution?

Meeting your needs for support and accommodations?

Creating an environment where this type of experience was safe to discuss?

Creating an environment where this type of experience was recognized as a problem?

Not doing enough to prevent this type of experience/s?

Creating an environment in which this type of experience/s seemed common or normal?

Creating an environment in which this experience seemed more likely to occur?

Making it difficult to report the experience/s?

Responding inadequately to the experience/s, if reported?

Mishandling your case, if disciplinary action was requested?

Covering up the experience/s?

Denying your experience/s in some way? 
Punishing you in some way for reporting the experience/s (e.g., loss of privileges or status)?

Suggesting your experience/s might affect the reputation of the institution?

Creating an environment where you no longer felt like a valued member of the institution?

Creating an environment where staying at your UC Library was difficult for you?

Responding differently to your experience/s based on your sexual orientation?

Creating an environment in which you felt discriminated against based on your sexual orientation?

Expressing a biased or negative attitude toward you and/or your experience/s based on your sexual orientation?

Responding differently to your experience/s based on your race?

Creating an environment in which you felt discriminated against based on your race?

Expressing a biased or negative attitude toward you and/or your experience/s based on your race? 


\section{Confidential}

$\begin{array}{ll}\text { Do you feel that your experiences of sexual } & \text { Yes } \\ \text { harassment in UC Libraries have affected your work? } & \text { No } \\ & \text { I'm not sure. } \\ & \text { OI prefer not to say. }\end{array}$

\begin{tabular}{ll}
\hline How important do you consider addressing sexual & O Very important \\
harassment in UC Libraries to be? & O Important \\
& $\bigcirc$ Moderately important \\
& O Slightly important \\
& O Not important
\end{tabular}

$\begin{array}{ll}\text { How important do you think your UC campus library } & \text { O Very important } \\ \text { administration considers addressing sexual } & \bigcirc \text { Important } \\ \text { harassment to be? } & \bigcirc \text { Moderately important } \\ & \bigcirc \text { Slightly important } \\ & \bigcirc \text { Not important }\end{array}$

How important do you think your UC campus administration considers addressing sexual

Very important harassment to be?

Important

Moderately important

Slightly important

Not important

What kind of steps or actions would you like to see

from UC Libraries or your campus library in

addressing sexual harassment?

\section{Confidential}

The following questions ask about demographic information and are optional. We recognize the importance of intersectionality when understanding experiences of sexual harassment; however, because of the lack of diversity in UC Libraries and the need to protect anonymity, we are unable to ask about some parts of your unique identity, including race and sexual orientation.

How do you identify your gender? Select all that apply. Please note: because of the importance of protecting anonymity of participants and the lack of diversity in UC Libraries, we are only able to collect information about broad gender identities.

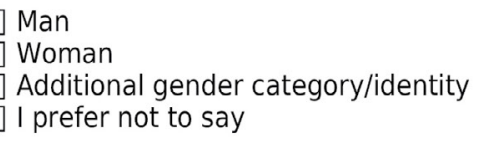

Please select your campus.
UC Berkeley
UC Davis
UC Irvine
O UCLA
UC Merced
$\bigcirc$ UC Riverside
$\bigcirc$ UC San Diego
UC San Francisco
U UC Santa Barbara
U UC Santa Cruz
I prefer not to say

Please include any additional thoughts or comments about the study here. 


\section{Confidential}

\section{Q16: Intersectionality \& sexual harassment}

This question is optional and will not be linked to your previous responses. Please do not provide any potentially identifying information (e.g. your name, campus, department, or specific details about people involved in any unreported incidents).

Survey results, including quotes from text provided in write-in sections, may be shared with UC Libraries staff, other members of the UC community, and within academic publications and presentations.

We are all members of different identity groups (e.g. gender, race, ethnicity, sexual orientation, age, socioeconomic class, faith/spiritual, type of role in the library). If there are parts of your identity that you feel affect your experiences of sexual harassment at UC Libraries, please describe them below so that we may become more aware of the diversity of library employees and intersectionality of experiences.

\section{Notes}

1. Jill Barr-Walker et al., "Sexual Harassment in the Library: Understanding Experiences and Taking Action," ACRL Proceedings (Apr. 2019): 421-27, www.ala.org/acrl/sites/ala.org.acrl/files/content/conferences/confsandpreconfs/2019/SexualHarassmentintheLibrary.pdf.

2. National Academies of Sciences, Engineering, and Medicine (NASEM), "Sexual Harassment of Women: Climate, Culture, and Consequences in Academic Sciences, Engineering, and Medicine" (2018), https://doi. org/10.17226/24994.

3. NASEM, "Sexual Harassment of Women."

4. Marina N. Rosenthal, Alec M. Smidt, and Jennifer J. Freyd, "Still Second Class: Sexual Harassment of Graduate Students," Psychology of Women Quarterly 40, no. 3 (2016): 364-77.

5. Candice Benjes-Small et al., "Quantifying the \#MeToo Narrative: Incidence and Prevalence of Sexual Harassment in Academic Libraries," ACRL Proceedings (Apr. 2019): 62-73, www.ala.org/acrl/sites/ala.org.acrl/ files/content/conferences/confsandpreconfs/2019/QuantifyingthemetooNarrative.pdf.

6. Kelly Jensen, "The State of Sexual Harassment in the Library," Book Riot (Oct. 2017). https://bookriot. com/2017/10/24/sexualharassment-library/; Amanda Civitello, “\#MeToo: Confronting Sexual Harassment in the Public Library," Public Libraries (Sept./Oct. 2017).

7. Jensen, "The State of Sexual Harassment in the Library."

8. Amanda Civitello and Katie McLain, "It's Not Just Part of the Job: Speaking Out about Sexual Harassment," ILA Reporter 6 (Nov. 2017): 4-7; Kelly Jensen, "Sexual Harassment in Libraries, Post-\#MeToo: What Has and Hasn't Changed?" Book Riot (Apr. 2019), https://bookriot.com/2019/04/08/sexual-harassment-in-libraries/.

9. University of California Office of the President, "Reliance MOU for IRB Review of Multi-Campus Human Subject Research" (Jul. 2016), https://www.ucop.edu/research-policy-analysis-coordination/policies-guidance/ human-subjects/multi-campus-human-subject-research-MOU.html.

10. University of California Office of the President Human Resources, "Policy on Sexual Harassment and Sexual Violence" (Jul. 2019), http://policy.ucop.edu/doc/4000385/SHSV.

11. Rosenthal, Smidt, and Freyd, "Still Second Class," 364-77.

12. Louise F. Fitzgerald et al., "The Incidence and Dimensions of Sexual Harassment in Academia and the Workplace," Journal of Vocational Behavior 32, no. 2 (Apr. 1988): 152-75.

13. Rosenthal, Smidt, and Freyd, "Still Second Class," 364-77. 
14. April Hathcock, "White Librarianship in Blackface: Diversity Initiatives in LIS," In the Library with the Lead Pipe (Oct. 2015); Jennifer Vinopal, “The Quest for Diversity in Library Staffing: From Awareness to Action," In the Library with the Lead Pipe (Jan. 2016).

15. Jill Barr-Walker et al., "Sexual Harassment at University of California Libraries: Understanding the Experiences of Staff Members" (2019), https://uclibraries.wixsite.com/shstudy.

16. Rosenthal, Smidt, and Freyd, "Still Second Class," 364-77.

17. University of California Sexual Violence Prevention and Response, "Education and Training" (April 7, 2020), https://sexualviolence.universityofcalifornia.edu/education-training/.

18. University of California Sexual Violence Prevention and Response, "Education and Training."

19. NASEM, "Sexual Harassment of Women," 23-50; Amir Karami et al., "Unwanted Advances in Higher Education: Uncovering Sexual Harassment Experiences in Academia with Text Mining," Information Processing E Management 57, no. 2 (Mar. 2020): 102167.

20. NASEM, "Sexual Harassment of Women," 23-50.

21. University of California Office of the President, "UC Undeterred Despite Harmful Federal Sexual Harassment Rules" (May 6, 2020), https://sexualviolence.universityofcalifornia.edu/education-training/.

22. Fitzgerald et al., "The Incidence and Dimensions of Sexual Harassment in Academia and the Workplace," $152-75$. 